EPJ manuscript No.

(will be inserted by the editor)

\title{
The one-dimensional Lennard-Jones system: collective fluctuations and breakdown of hydrodynamics
}

\author{
Stefano Lepri ${ }^{\text {a }}$, Paolo Sandri, and Antonio Politi \\ Istituto dei Sistemi Complessi, Consiglio Nazionale delle Ricerche, Sez. Territoriale di Firenze \\ ' and Istituto Nazionale di Ottica Applicata, largo E. Fermi 6 I-50125 Firenze, Italy
}

Received: August 24, 2018

\begin{abstract}
The dynamical correlations of a model consisting of particles constrained on the line and interacting with a nearest-neighbour Lennard-Jones potential are computed by molecular-dynamics simulations. A drastic qualitative change of the spectral shape, from a phonon-like to a diffusive form, is observed upon reducing the particle density even ad moderate temperatures. The latter scenario is due to the spontaneus fragmentation of the crystal-like structure into an ensemble of "clusters" colliding among themselves. In both cases, the spectral linewidths do not follow the usual $q^{2}$ behaviour for small wavenumbers $q$, thus signalling a breakdown of linearized hydrodynamics. This anomaly is traced back by the presence of correlations due to the reduced dimensionality.
\end{abstract}

PACS. 05.60.-k Transport processes - 66.10.Cb Diffusion and thermal diffusion

\section{Introduction}

Relaxation and transport phenomena in reduced spatial dimension $(D<3)$ are often qualitatively different from their three-dimensional counterparts. For concreteness, imag-Tl ine a large set of impenetrable spheres confined within a narrow channel. If the mutual passage of particles is forbidden, the motion of the spheres is necessarily correlated, even at long times, because the displacement of a given particle over a long distance necessitates the motion of many other particles in the same direction. This is a documented effect, for example, in single-filing systems where particle diffusion does not follow Fick's law [1,2. Another related instance is the enhancement of vibrational energy transmission in quasi- $1 D$ systems like polymers [3] or individual carbon nanotubes [4].

The distinguished signature of those effects is in the long-time behavior of the associated correlation functions [5]. As it is known, the latter may display long-time tails leading to ill-defined transport coefficients or, more generally, to the breakdown of customary hydrodynamics. Indeed, power-law decay of correlations is expected to be a generic feature of one-dimensional systems in presence of conservation laws [6]. One important consequence of such long-ranged correlations is that physical properties may significantly depend on the system size and that the thermodynamic and infinite-time limits may not commute. For instance, the tagged-particle diffusion coefficient in $D=1$, that is finite for the infinite system is found instead

\footnotetext{
a Electronic address: stefano.lepri@isc.cnr.it
}

to vanish for a finite one (see [7] and references therein). Another example is the divergence of the thermal conductivity coefficient with the length observed in chains of anharmonic oscillators [8, 9] and hard-points gas [10]. -The same type of anomaly has been detected also for a quasi $-1 D$ model consisting of spheres confined in a narrow channel [11.

Computer simulations of simple toy models is an invaluable way of attacking those problems. In particular, one would like to understand the conditions under which those anomalies occur and possibly to classify the possible universal features. In this paper we consider a simple model of point particles constrained on the line and interacting with their nearest neighbours through a LennardJones force. This type of phenomenological interaction has been throughly studied for decades by molecular-dynamics methods [12]. However its one-dimensional version has received little attention so far. Previous studies focused on anharmonic effects 13,14 and transport properties [15]. In this respect, some evidence that the energy current autocorrelation (the Green-Kubo integrand) shows a longtime tail of the above mentioned type has been provided too 16. The same system has been also proposed as a toy model to describe fracture nucleation [17,18.

Of particular relevance in what follows is the work of Bishop and collaborators [19 20. At variance with our model, they considered the case in which the interaction is extended to all particle pairs. They noticed that the lifetime of long-wavelength fluctuations does not scale with their wavenumber $q$ as $q^{-2}$, as expected from standard 
Stefano Lepri et al.: The one-dimensional Lennard-Jones system

hydrodynamics, but rather as a nontrivial power $q^{-\mu}$. We anticipate that the results presented henceforth are qualitatively consistent with theirs. However, the estimate of the exponent $\mu=1 / 3$ given in Ref. 20 is significantly different from the value found here, $\mu \simeq 1.5$, which is consistent with previous measurements in chains of coupled oscillators 21,22]. This issue is relevant especially for assessments about the universality of the scaling laws.

The present paper is organized as follows. In section 2 we define the model and its physical parameters. Section 3 is devoted to a discussion of the nature of the ground state at zero temparature. This is important to understand the effect of density changes on the system dynamics. In Section 4 we present our simulation results and show how the low-density properties are related to the kinetics of particle clusters. Finally, we summarize our results in the concluding Section.

\section{The model}

We consider an array of $N$ point-like identical atoms ordered along a line. The position of the $n$-th atom is denoted with $x_{n}$. By fixing the mass, without loss of generality, equal to unity and assuming that interactions are restricted to nearest-neighbour pairs, the equations of motion write

$$
\ddot{x}_{n}=-F_{n}+F_{n-1} \quad ; \quad F_{n}=-V^{\prime}\left(x_{n+1}-x_{n}\right),
$$

where $V^{\prime}(z)$ is a shorthand notation for the first derivative of the the interparticle potential $V$ with respect to $z$. The particles are confined in a simulation "box" of length $L$ with periodic boundary conditions

$$
x_{n+N}=x_{n}+L \text {. }
$$

Accordingly, the particle density $d=N / L$ is a state variable to be considered together with the specific energy (energy per particle) that will be denoted by $e$.

In the present work we focus on the Lennard-Jones potential that in our units reads

$$
V(z)=\frac{1}{12}\left(\frac{1}{z^{12}}-\frac{2}{z^{6}}+1\right) .
$$

For computational purposes, the coupling parameters have been fixed in such a way as to yield the simplest form for the force. With this choice, $V$ has a minimum in $z=1$ and the resulting dissociation energy is $V_{0}=1 / 12$. Notice that for convenience we set the zero of the potential energy in $z=1$. The presence of the repulsive term in one dimension ensures that the ordering is preserved (the particles do not cross each other).

Before closing this section, let us recall that most previous studies [16, 15, 19,20 dealt with the case in which the interaction is not restricted to nearest neighbours but rather extends all particle pairs. In practice, as pointed out in Refs. 19 20, even in this case the interaction is limited to about 2-3 neighbours. Therefore, we do not expect that the results reported below will be significantly altered when taking into account the interaction among all pairs.

\section{The ground state at $T=0$}

In order to understand the physical features of the model let us briefly discuss its equilibrium properties. Since the system is one dimensional with only nearest-neighbour and short-ranged interaction, no actual phase transition at finite temperature, $T>0$, can occur. Nevertheless, we will see that the dynamics of collective modes may strongly depend on the state variables. Therefore, even if it is not legitimate to speak of "phases" in a strict thermodynamic sense, it is sensible to distinguish between regions where the dynamics is qualitatively different.

Let us start discussing the equilibrium configurations at $T=0$ as a function of the particle density $d$. This issue, which is important for the choice of the initial conditions in microcanonical simulations (see below), has been discussed in Ref. 23. for the Lennard-Jones chain where the pair interaction is among all particles. In view of the shortrange nature of the potential we do not find significative difference with the case at hand here. For convenience, we summarize the main results in Fig 1. Three density regimes are distinguished:

- For $d \geq 1$ the ground state consists of equally spaced particles at a relative distance $a=1 / d$ (homogeneous solution). The chain is under compression and the total energy decreases upon decreasing $d$ up to the minimum value which is attained for $d=1$.

- For $d_{*}<d<1$ the homogeneous solution (chain under tension) becomes a relative minimum. The ground state consists of equally spaced particles ad a distance approximatively $a \approx 1$ except for a couple which lies at a large distance. In other words, the minimal energy configuration is attained by breaking the chain at one bond. At the critical value $d=d_{*}=\sqrt[6]{\frac{7}{13}}=$ $0.901971 \ldots$, the homogeneous configuration undergoes an instability and disappears.

- For $d<d_{*}$ the broken chain state is the unique minimal energy configuration.

Notice that for $d<1$ the ground-state energy is basically 0 up to terms $\mathcal{O}(1 / N)$ since only a couple of particles out of $N$ sits at a distance which is significantly larger than 1. Furthermore, as noted in Ref. [23], further fragmentation in two or more shorter chains cannot produce further energy minima.

The phase diagram of Fig. 11 suggests a first-order phase transition at $d=1$ where the second derivative of the energy density with respect to $d$ undergoes a jump discontinuity. As this quantity should be proportional to the Young's modulus this physically means that the chain undergoes a fracture and looses its elastic tension.

At finite temperature we expect that these features should be washed out but, as observed for other models 24], the remnants of the transition should somehow manifest themselves in the dynamics. 


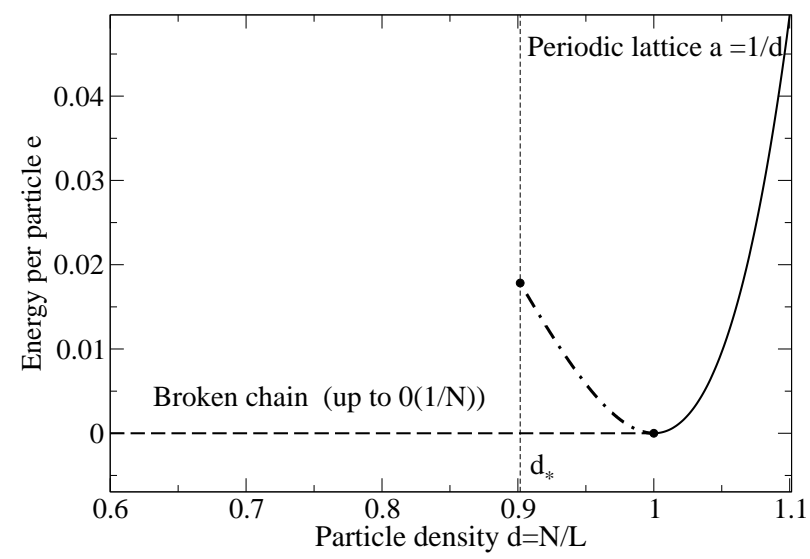

Fig. 1. The $T=0$ minimal-energy configurations of the Lennard--Jones chain as a function of the particle density.

\section{Dynamical correlations}

We have performed equilibrium microcanonical simulations by integrating Eqs. (11) (with periodic boundary conditions (2) ) by means of a fourth-order symplectic algorithm [25. For the different values of density considered henceforth, initial conditions were chosen to be in the ground state described in the previous section. The inital velocities were drawn at random from a Gaussian distribution and rescaled by suitable factors to assign the kinetic energy to the desired value and to set the total initial momentum equal to zero. A suitable transient is elapsed before acquisition of statistical averages. Conservation of energy and momentum was monitored during each run. This check is particularly crucial at high energies/densities where the strongly repulsive part of the force comes into play and may lead to significant inaccuracies. The chosen time-step (0.005-0.05) ensures energy conservation up to a few parts per million in the worst case.

We computed the dynamical structure factor, namely the square modulus of temporal Fourier transform of the particle density

$$
\rho(q, t)=\frac{1}{N} \sum_{n} \exp \left(-i q x_{n}\right)
$$

which is defined as

$$
S(q, \omega)=\left\langle|\rho(q, \omega)|^{2}\right\rangle
$$

The square brackets denote an average over a set of independent molecular-dynamics runs. By virtue of the periodic boundaries, the allowed values of the wavenumber $q$ are integer multiples of $2 \pi / L$. The reliability of the spectra have been checked against different choices of the run durations and sampling times. In some particular cases we also verified that the results are not affected by data windowing that, in principle, may affect the measured linewidths.

\subsection{The $d=1$ case}

In this case we expect a "lattice-like" behaviour with each particle oscillating around its equilibrium positions. In other words, we can introduce the change of coordinates $x_{n}=u_{n}+n a$ where $a=1 / d$ is the constant lattice spacing. The computation of $S(q, \omega)$ can be more conveniently performed by resorting to the collective coordinates

$$
U(q)=\frac{1}{\sqrt{N}} \sum_{n=1}^{N} u_{n} \exp (-i q n) \quad q=\frac{2 \pi k}{N a}
$$

for $k$ being an integer comprised between $-N / 2+1$ and $N / 2$. This latter expression is computationally more efficient than the definition (4). In fact, standard Fast Fourier Transform routine can be used to evaluate $U(q)$ (provided that $N$ is a power of 2 ). On the other hand, by expanding to the leading order in $q$, it is seen that the structure function (5) is proportional to $q^{2}\left\langle|U(q)|^{2}\right\rangle$. We checked numerically that this approximation is very accurate in the range of $q$ values considered henceforth.

In Fig. 2 we report two representative data sets for low $(e=0.02)$ and high energies $(e=0.2)$ compared with the well depth. The most distinguished feature of the the spectra is a narrow phonon peak. For the low energy case, its frequency $\Omega$ agrees with the one computed by the harmonic approximation of the Lennard-Jones potential:

$$
\Omega(q)=2 \sqrt{V^{\prime \prime}(1)}\left|\sin \frac{q a}{2}\right| .
$$

Indeed, the estimated sound speed $c=2.77 \pm 0.06$ is only slightly larger the value obtained from this latter expression $(c=\sqrt{6} \approx 2.49 \ldots)$. This indicated that nonlinear terms are weak in this energy range. At higher energies, the particles are less sensitive to the bottom part of the potential well and the phonon peaks shifts to higher frequency. The data in Fig. 2 b correspond to $e=0.2$, more than 20 times the well depth; comparing the values of Fig. 23 it can be ascertained that the frequencies are roughly $50 \%$ larger than in the previous case.

For small enough wavenumbers, besides the phonon peak, a small zero-frequency component appears whose relative weight increases at larger energies (see again Fig. 2). This feature, that has not been reported in previous works on the Lennard-Jones chain [13], is reminiscent of the wellknown triplet structure observed in fluids where a central Rayleigh peak is accompanied by two narrow Brillouin lines placed at $\pm c q$ [26]. Moreover, according to the standard hydrodynamic theory, the ratio of the areas under half the Rayleigh peak and one Brillouin peak is $C_{p} / C_{V}-1$. On the other hand, the specific heats ratio is expected to be very close to unity for a "crystal-like" structure as the one we are facing in our simulation. This is thus consistent with the intuitive idea that a sizeable central component should occur only when the systems is "fluid enough" i.e. when fluctuations of the particles' positions are large. This is expected for large temperatures and/or small densities ${ }^{1}$. In the next section we will see

\footnotetext{
1 The existence of a central component also means that there is a coupling between density and energy fluctuations which are
} 
that this expectation is further confirmed by the simulations.
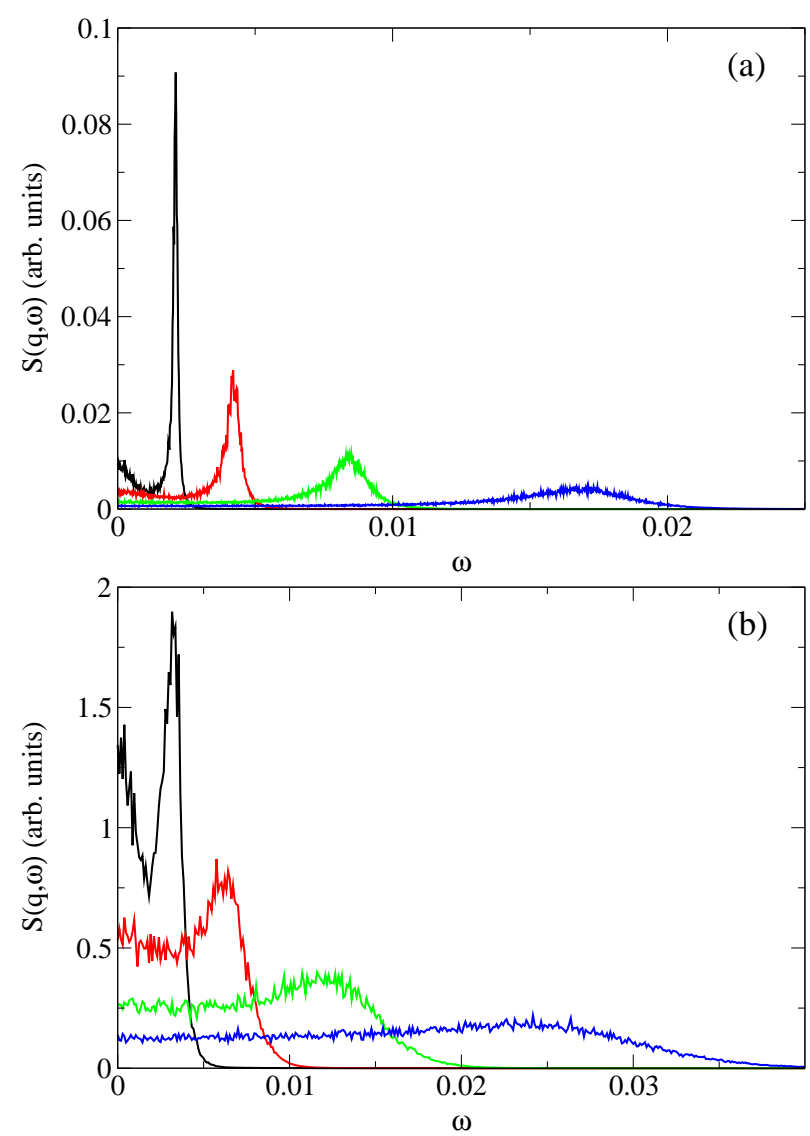

Fig. 2. Structure factors for $d=1, N=8192$ and different $q \mathrm{~s}$ corresponding to indices $k=1,2,4,8$ in eq. (6) (left to right). Microcanonical simulations are performed for the energy densities $e=0.02$ (a) and $e=0.2$ (b). The spectra are averaged over an ensemble of about 100 initial conditions.

In Fig. 3we report the linewidths of the Brillouin peaks as a function of the wavenumber $q$ and for the two energy values chosen above. The data reported there correspond to those wavenumbers for which the linewithds are small enough with respect to the peak frequency (typically less the one tenth) to allow for a meanigful estimate. The linewidths are computed by evaluating the frequencies at which the spectrum is one half of its maximal value. In the cases in which the spectral resolution is not high enough we used by a Lorenzian fit to improve the accuracy. Incidentally, we noticed that the the fitting is reasonably good only in the peak region while substantial deviations on the tails are observed.

Remarkably, the linewidths do not scale as $q^{2}$ as expected from standard hydrodynamics but rather as $q^{\mu}$. For $e=0.02$ our best-fit estimate is $\mu=1.47 \pm 0.05$. To

the hydrodynamic fields of our model. To support this interpretation we computed also the spectra of the local energy field in some cases. They do display a similar structure with a large component at the phonon frequency. better appreciate the reliability of this value, in the inset of Fig. 3 we report the logarithmic derivative of the data that show a plateau around 1.5. The data for $e=0.2$ are instead less conclusive. Although a power law fit still yields a comparable value $(\mu=1.41 \pm 0.07)$ the plot displays a residual curvature that indicate some relevant subleading corrections.

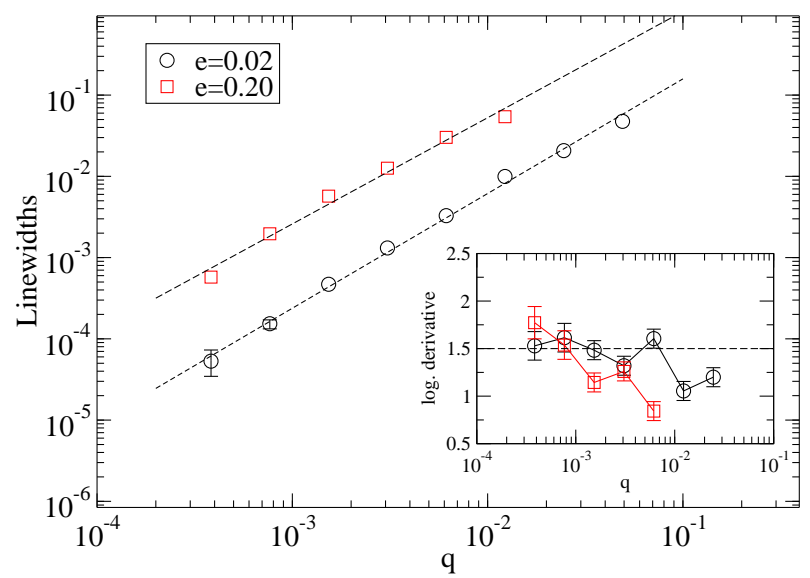

Fig. 3. The linewidths of the phonon peak for $d=1, e=0.02$ and $e=0.2$. Error bars are reported only when significantly larger than the symbols. The inset shows the logarithimc derivative evaluated by finite differences.

\subsection{The $d<1$ case}

At finite temperature, when the density is lowered below $d=1$, the periodic ground state destabilizes and this leads to a completely different dynamics. To avoid the additional features connected with the presence of the metastable branch (see Fig. 10 we consider the case $d<d_{*}$. Fig. 4 shows the evolution of the particle density field starting from the minimal-energy configuration for $d=0.8$. After a transient, the system spontaneously fragments in a series of clusters of different lengths. Within each cluster, the particles are separated by an average distance equal to the equilibrium distance of the LennardJones potential ( 1 in our units).

The relaxation is thus ruled by the clusters' kinetics. The latter consists of two different processes, fragmentation and collisions whose characteristic times we denote by $\tau_{F}$ and $\tau_{C}$ respectively. Let us start giving an estimate of the average number $m$ of particles in each cluster. For convenience, we write $m=1 / f$ where $f$ is the average fraction of broken bonds. The average time between collisions is given by the ratio between the typical clusters' separation and the typical speed $v$. A straightforward calculation yields

$$
\tau_{C}=\frac{1}{v f}\left(\frac{1}{d}-1\right)
$$

(remember that the equilibrium distance is 1 in our units). On the other hand, we expect fragmentation to be a thermally activated process with an activation energy equal 


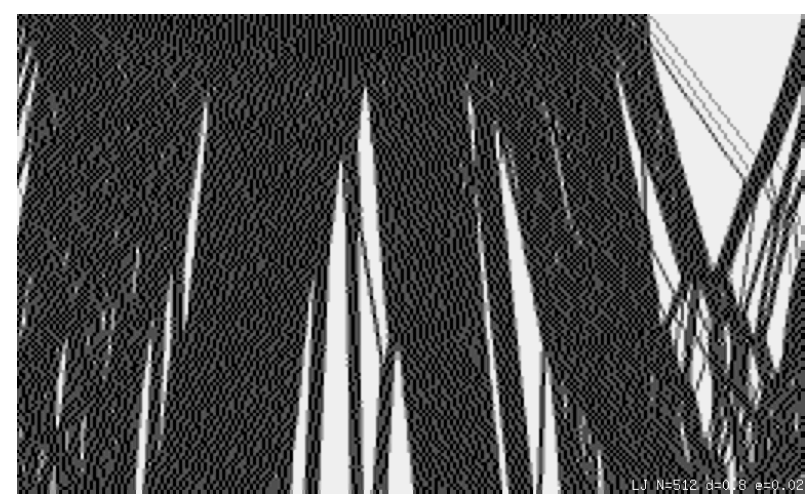

Fig. 4. The evolution of the particle density for $d=0.8, N=$ 512 and $e=0.02$. Along the horizontal direction a black pixel is drawn at each particle location. Time increases downwards.

to the well depth $V_{0}$. Accordingly,

$$
\tau_{F}=\tau f \exp \beta V_{0},
$$

where $\tau$ is a suitable prefactor setting the typical "attempt time" for the process and $\beta$ is the inverse temperature. The condition for kinetic equilibrium is obtained by letting $\tau_{C}=\tau_{F}$,

$$
f=\sqrt{\frac{1-d}{\tau v d}} \exp \left(-\frac{\beta V_{0}}{2}\right) .
$$

Notice that, as a consequence of this estimate, the characteristic time is proportional to $\sqrt{1 / d-1} \exp \left(\beta V_{0} / 2\right)$ and that thermalization may become very slow for low temperatures and densities.

This prediction is in fairly good agreement with the numerical data (Fig. 5). There, we plot the average fraction of broken bonds $f$ as a function of the inverse kinetic temperature. The quantity $f$ is measured by counting at each time the number of pairs whose distance is larger than some prescribed threshold that we fixed equal to 1.5 (some $50 \%$ above the inflection point of the Lennard-Jones potential). From the above discussion, it is clear that the choice of well-thermalized initial conditions is crucial. Indeed we found that the time to reach the equilibrium value of $f$ increases (about linearly) with the number of particles. In Fig. 5 it is shown that $f$ is an extensive parameter whose equilibrium value can be evaluated already for systems of a few hundred particles. In Fig. 15 we check that the scaling behaviour predicted by the kinetic argument reported above is in agreement with the simulation data. Notice in particular that the factor $1 / 2$ in the exponential term is very well accounted for by the data and that the prefactors display a rather weak dependence on $\beta$.

In view of the above dynamical features, we expect that the the spectra of density fluctuations should be qualitatively different from the $d=1$ case. This is confirmed by the simulation reported in Figs. [6] (in this low-density regime we use the definition (5) of $S(q, \omega)$ ). Indeed, even at low energies, a large central component appears which we associate with the diffusive behaviour of the clusters. The secondary Brillouin peaks occur only for small enough
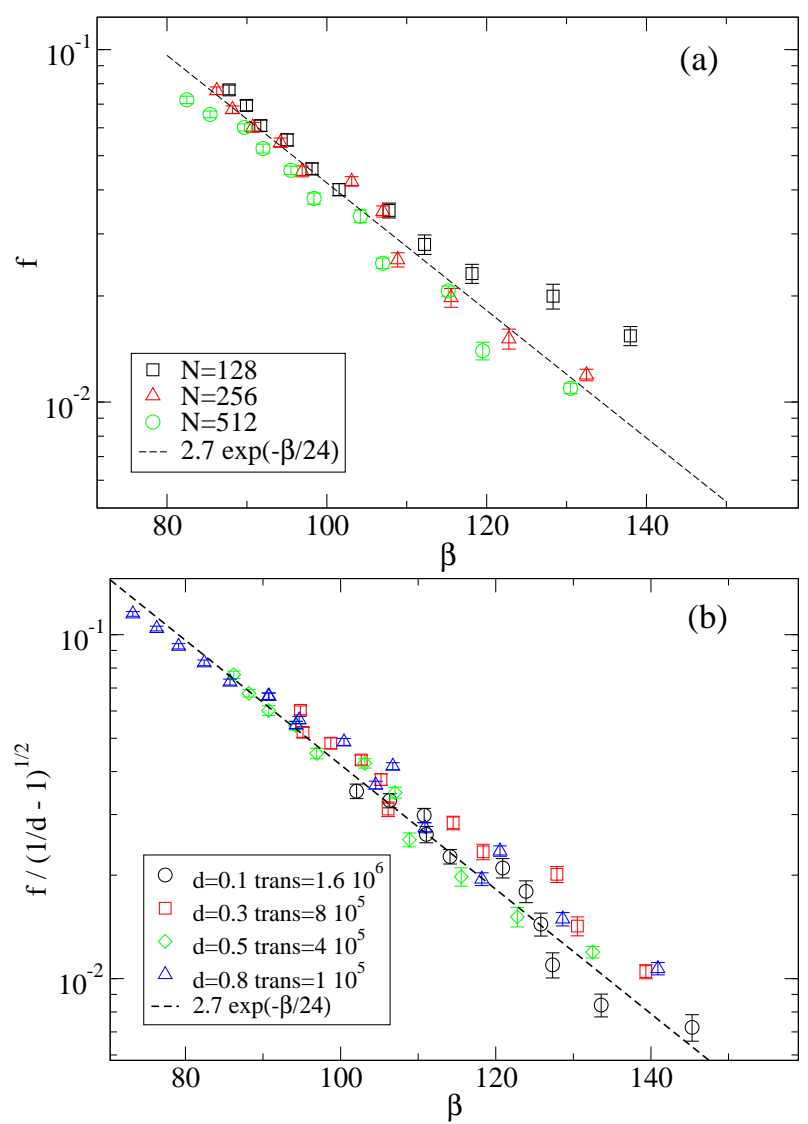

Fig. 5. The average fraction of broken bonds $f$ as a function of the inverse kinetic temperature $\beta$; (a) dependence of $f$ on the number of particles $N$ for fixed density $d=0.5$; (b) check of the formula (10) for fixed $N=256$.

wavenumbers and are weaker: for the smallest wavenumber shown in Fig. 6 the secondary peak has about $40 \%$ spectral power of the central component. This observation is consistent with the intuitive expectation that at lower densities the system should respond like a fluid.

The behavior for $e=0.2$ is shown in Fig. 6b. The structure factor is qualitatively similar to the case of Fig. $2 \mathrm{~b}$. This is expected, since in the high energy limit the model approach the case of hard points colliding elastically. It is thus plausible that the dynamics is practically independendent on the particle density. Notice also that in this limit the model becomes an almost-integrable dynamical system and some some pathological behaviour (e.g. slow thermalization) may be expected.

In analogy with the case $d=1$ we analysed the dependence of the spectral linewidths on the wavenumber, considering only those $q$ values that correspond to narrow enough lines. Fig 7 shows the half-widths of the central (Rayleigh) peak as a function of the wavenumber $q$ for the $e=0.02$. In the accessible range we find again a nontrivial scaling $q^{\mu}$ with $\mu=1.60 \pm 0.05$. 

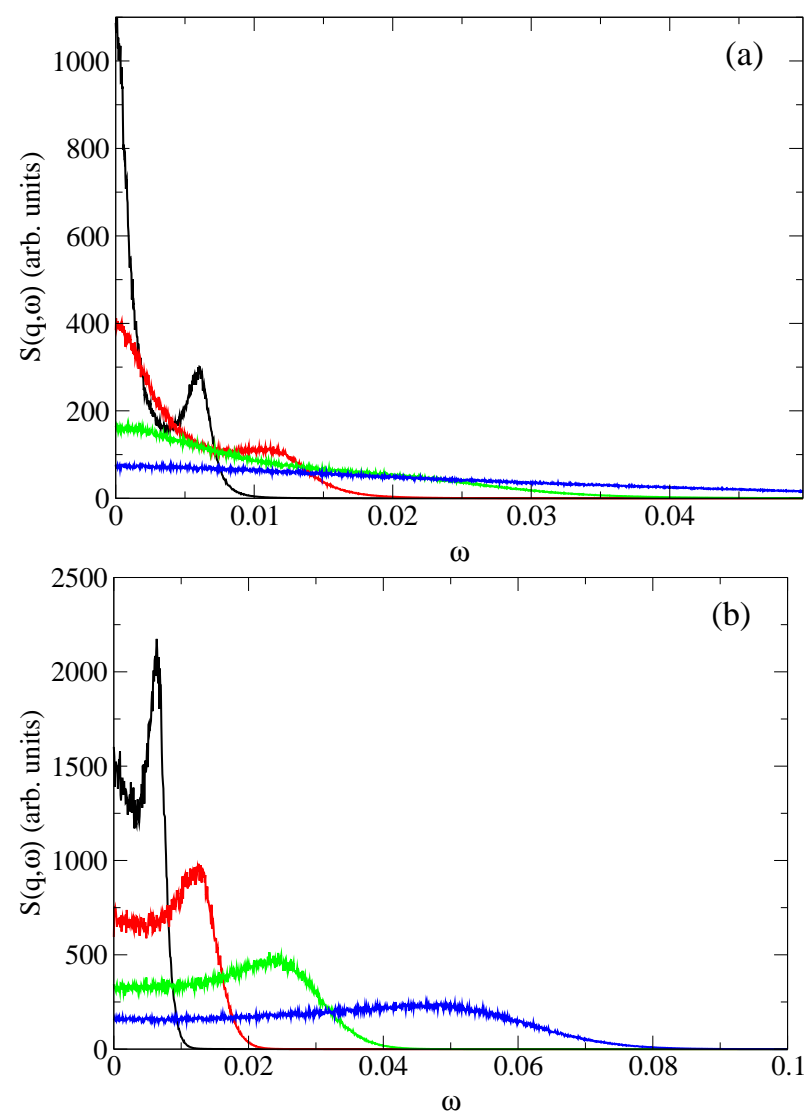

Fig. 6. Structure factors for $d=0.8$ and different $q$ s corresponding to indices $k=1,2,4,8$ in eq. (6) (left to right). Microcanonical simulations are performed for the energy densities $e=0.02, N=1024$ (a) and $e=0.2, N=4096$ (b). The spectra are averaged over an ensemble of about 500 and 100 initial conditions respectively.

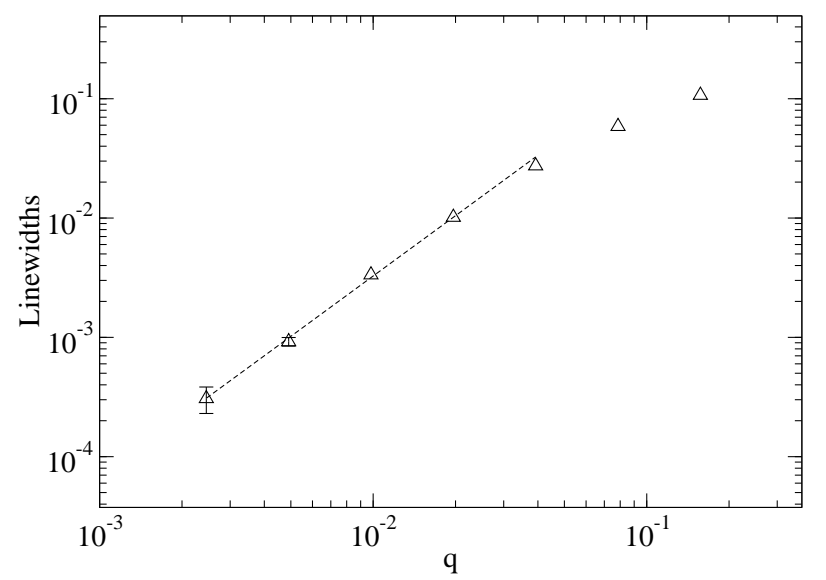

Fig. 7. The linewidths of the central peak for $d=0.8$ and $e=0.02$. Error bars are reported only when significantly larger than the symbols.

\section{Conclusions}

Our numerical analysis of the one-dimensional LennardJones system has shown that long-ranged correlations induced by the reduced spatial dimensionality may signif- icantly affect the density fluctuations. The most striking feature is the anomalous scaling of the Rayleigh and Brillouin peak widths in the hydrodynamic limit, $q \rightarrow 0$. The physical meaning of this behaviour is understood by recalling that, in the standard framework, the linewidths are connected to transport coefficients 26. For instance the width of the Brillouin peak is $\Gamma q^{2}$ where $\Gamma$ is the sound attenuation constant. The anomalous scaling can be recasted in terms of a wavenumber-dependent constant $\Gamma(q) \sim q^{\mu-2}$. Since our simulations clearly indicate that $\mu<2$, this implies that $\Gamma$ diverges in the $q \rightarrow 0$ limit. In other words, a long-wavelength disturbance is damped on a typical distance which becomes very large. In some sense, one may think of this as a superdiffusive process, intermediate between standard diffusive and ballistic propagation. Our result is thus closely related to the analysis performed in Ref. 27] for the hard-point gas.

This feature has been previously observed also in other one-dimensional lattice models [21,22]. A similar anomalous behaviour of the viscosity of a $1 D$ lattice gas has also been reported in Ref. 28. However, the exponent $\mu$ found in the present work is about $10 \%$ smaller then those measured in Refs. 21.22 (1.5 against 1.67). Remarkably, both values are very close to theoretical estimates presented previously in the literature. Ernst 29 claimed that $\mu=5 / 3$, while the more refined mode-coupling analysis by Wang and $\mathrm{Li}$ 30 gave $\mu=3 / 2$ for the specific model they considered. We anticipate that this latter value is actually supported by numerical solution of the modecoupling equations 31] that, according to Ref. 14, should describe the dynamical properties of our model. This estimate is in excellent agreement with the data reported above. Whether the small differences in the exponents are due to numerical errors and subleading corrections or they indicate the existence of two different "universality classes" is still an open question.

For $d=1$ the system retains the dynamical features of a one-dimensional crystal. Of course, by virtue of the short-ranged interactions, the model cannot have a genuine solid phase (Landau-Peierls instability). Nonetheless, we have found that for a finite system we can still reason in terms of an effective phonon dispersion and damping. In the low-density region $(d<1)$ we have shown how the relevant time scales are instead dictated by the collision and recombination processes of one-dimensional "clusters" of particles. Such processes may lead to a remarkable increase of the thermalization times when the kinetic temperature becomes smaller than the binding energy (see Eqs. (9 and 10).

In the present study we limited ourselves to the case in which the system is initialized close to the ground state. It would be interesting to examine the relaxation from the metastable state which exists for $d_{*}<d<1$. In this respect there is a connection with the work of Oliveira [18] that studied the fracture nucleation in the same model. At variance with what discussed here, he starts from the uniformly stretched chain, namely from the metastable state illustrated in the phase diagram of Fig. 1 He founds that the breaking time is orders of magnitude longer than what 
expected from Kramers-type estimate. This is presumably to be traced back to subtle long-ranged correlations of the very same type studied here.

To conclude, we wish to mention the fact that the anomalous scaling may manifest also at the level of energy transport in this model. Indeed, nonequilibrium simulations show that the thermal conductivity coefficient diverges with the system length also in the low-density regime 32 . This is a further evidence that nonequilibrium processes in one dimension are peculiar and deserve a special attention.

\section{Acknowledgments}

This work is supported by the PRIN2003 project Order and chaos in nonlinear extended systems funded by MIUR-Italy. Part of the numerical simulation were performed at CINECA supercomputing facility through the INFM Iniziativa trasversale "Calcolo Parallelo" entitled Simulating energy transport in low-dimensional systems.

\section{References}

1. K. Hahn, J. Kärger, and V. Kukla, Phys. Rev. Lett. 76, 2762 (1996).

2. Q.-H. Wei, C. Bechinger, P. Leiderer, Science 287, 625 (2000).

3. D.T. Morelli, J. Heremans, M. Sakamoto, C. Uher, Phys. Rev. Lett. 57, 869 (1986).

4. J. Hone et al., Phys. Rev. B 59 (1999) R2514; S. Maruyama, Physica B 323 (2002) 193.

5. Y. Pomeau, R. Résibois, Phys. Rep. 19 (1975) 63.

6. O. Narayan, S. Ramaswamy, Phys. Rev. Lett. 89, 200601 (2002).

7. S. Pal, G. Srinivas, S. Bhattacharyya and B. Bagchi, J. Chem. Phys. 1165941 (2002).

8. S. Lepri, R. Livi, A. Politi, Phys. Rev. Lett. 78, 1896 (1997); Europhys. Lett. 43, 271 (1998).

9. S. Lepri, R. Livi, A. Politi, Phys. Rep. 377, 1 (2003).

10. T. Hatano, Phys. Rev. E 59, R1 (1999); P. Grassberger, W. Nadler, L. Yang, Phys. Rev. Lett. 89, 180601 (2002).

11. J.M. Deutsch, O. Narayan, Phys. Rev. E 68, 010201 (2003).

12. D. Frenkel, B. Smit, Understanding Molecular Simulation: from Algorithms to Applications (Academic Press, 1998).

13. A. Cuccoli, V. Tognetti, A. A. Maradudin, A. R. McGurn and R. Vaia, Phys. Rev. B 48, 7015 (1993).

14. J. Scheipers, W. Schirmacher, Z. Phys. B 103547 (1997).

15. A. M. Bazhenov, D.M. Heyes, J. Chem. Phys. 921106 (1990).

16. M. Mareschal, A. Amellal, Phys. Rev. A 37, 2189 (1988).

17. R.W. Welland, M. Shin, D. Allen and J.B. Ketterson, Phys. Rev. B 46, 503 (1992).

18. F. A. Oliveira, Phys. Rev. B 57, 10576 (1998).

19. M. Bishop, M. Derosa and J. Lalli, J. Stat. Phys 25 (2), 229 (1980).

20. M. Bishop, J. Stat. Phys 29 (3), 623 (1982).

21. S. Lepri, Phys. Rev. E 587165 (1998).

22. S. Lepri, R. Livi, A. Politi, Chaos 15, 015118 (2005)
23. F.H. Stillinger, Phys. Rev. E 52, 4685 (1995).

24. S. Flach and J. Siewert, Phys. Rev. B 4714910 (1993).

25. R.I. Mclachlan, P. Atela, Nonlinearity 5, 541 (1992).

26. D. Forster, Hydrodynamic Fluctuations, Broken symmetry and Correlation Functions (W. A. Benjamin, Reading, 1975).

27. P. Cipriani, S. Denisov and A. Politi, Phys. Rev. Lett. 94, 244301 (2005) .

28. D. D'Humieres, P. Lallemand, Y. Qian, C. R. Acad. Sci. Paris 308, serie II 585(1989).

29. M.H. Ernst, Physica D 47198 (1991).

30. J. S. Wang and B. Li, Phys. Rev. Lett. 92, 074302 (2004); Phys. Rev. E 70, 021204 (2004)

31. L. Delfini, S. Lepri, R. Livi and A. Politi (unpublished).

32. P. Cipriani, private communication. 